\title{
PERCEPÇÕES DOS ESTUDANTES DE ENGENHARIA ACERCA DO LIVRO DE CÁLCULO DIFERENCIAL E INTEGRAL
}

DOI: 10.37702/2175-957X.COBENGE.2021.3634

Lino Marcos da Silva - lino.silva@univasf.edu.br

Univasf

Rua Vital Brasil 158

56330-260 - Petrolina - PE

Andressa da Silva Lino - lino.andressa97@gmail.com

Univasf

Rua do Cajueiro 912

48904-083 - Juazeiro - BA

Geida Maria Cavalcanti de Sousa - mmm@dfdfd

Univasf

Rua Pau Ferro 310

56306-100 - Petrolina - PE

Resumo: O livro didático é um importante instrumento no processo de ensino e aprendizagem de uma disciplina. Em particular, na disciplina Cálculo Diferencial e Integral, este instrumento tem grande relevância por ser um importante interlocutor entre o conteúdo da disciplina e o aluno. Contudo, sabe-se que a escolha dos livros didáticos é, em geral, realizada apenas sob a ótica do professor da disciplina. Dessa maneira, é possível que elementos valorizados pelos discentes e que contribuem efetivamente com sua aprendizagem não estejam sendo considerados nos livros de cálculo adotados nos cursos de engenharia, deixando assim de cumprir o seu papel. Neste trabalho, utilizou-se de uma pesquisa de campo, de cunho qualiquantitativo, cuja investigação pretendeu analisar um fato social, o uso do livro didático de Cálculo adotado nos cursos de engenharia, com vistas a elaborar um panorama do livro na visão dos alunos que cursaram a disciplina numa Universidade Federal. Os principais resultados obtidos apontam que o livro de cálculo pode ter uma influência direta na aprovação dos alunos na disciplina, que a clareza da linguagem utilizada pelos autores é um elemento muito relevante para o aprendizado dos alunos e que estes sugerem melhorias na linguagem, na quantidade de exemplos, de exercícios contextualizados, de exercícios resolvidos e de respostas aos exercícios propostos nos livros-texto. 


\section{COBENGE

Palavras-chave: Ensino de Matemática, Cálculo Diferencial e Integral, Livro Didático. 


\section{PERCEPÇÕES DOS ESTUDANTES DE ENGENHARIA ACERCA DO LIVRO DE CÁLCULO DIFERENCIAL E INTEGRAL}

\section{INTRODUÇÃO}

A disciplina Cálculo Diferencial e Integral é obrigatória nos cursos de bacharelado em Engenharia e se constitui, frequentemente, no primeiro contato do aluno egresso do Ensino Médio com a Matemática do Ensino Superior. Nessa etapa do ensino, é razoável intuir que os conceitos matemáticos são mais avançados e, quase sempre, são abordados com mais formalismo e rigor teórico. No entanto, essa mudança na postura de abordagem dos conteúdos em sala de aula, aliada à baixa proficiência por parte da maioria dos alunos ingressantes no ensino superior, tem implicações sérias nos cursos que possuem a disciplina Cálculo Diferencial e Integral, a exemplo do caso das engenharias com elevados índices de reprovação, retenção e abandono.

O elevado índice de reprovação na disciplina, que é considerado alto e parece oscilar, no mesmo patamar, nas diversas instituições que ofertam cursos de Engenharia no país, tem sido estudado ao longo dos anos. Os resultados apontam que são diversas as variáveis que potencialmente contribuem para esse fenômeno, como por exemplo, metodologia docente (PASSOS et al., 2007b); falta de estudo, dificuldade de acesso ao acervo bibliográfico e falta de monitoria (PASSOS et al., 2007a), deficiência na formação básica dos alunos (MENDES e GIOSTRI, 2008) e dificuldades conceituais (CURI e FARIAS, 2008).

Apesar de os estudos citados terem lançado uma luz sobre a problemática em questão, o assunto é bastante complexo e a realização de mais estudos e análises sobre diferentes enfoques devem ser realizados, de modo a possibilitar uma compreensão global do problema e, por conseguinte, melhores chances de construção de soluções efetivas para sua mitigação.

No contexto do ensino e aprendizagem do Cálculo Diferencial e Integral, o livro didático é um recurso bastante utilizado por professores e estudantes. No entanto, apesar das ementas e programas da disciplina apresentarem, em geral, uma variedade de títulos, a escolha do livro tem sido realizada exclusivamente sob a perspectiva do professor. Dessa maneira, é pertinente questionar se os livros didáticos adotados têm atendido as necessidades de aprendizagem dos estudantes e qual é a percepção dos estudantes sobre as principais obras adotadas em seus cursos e indicadas por seus professores.

Num contexto mais geral, conforme afirmado na II Conferência Internacional em Pesquisa e Desenvolvimento de Livros didáticos de Matemática (ICMT2), realizada no Rio de Janeiro no ano de 2017, a investigação com foco na análise e no desenvolvimento de livros didáticos vem ganhando destaque no cenário internacional da pesquisa em educação matemática, sendo a realização do evento uma prova disso. No caso específico do livro de Cálculo, vários estudos têm sido realizados nos últimos anos, utilizando-se de diferentes abordagens e proporcionando diferentes pontos de vistas sobre o tema. Dentre eles, destacam-se: uma investigação sobre como a organização didática e matemática dos livros textos interferem no processo ensino e aprendizagem da disciplina (MATEUS, 2007); a investigação sobre a transposição didática de conteúdos em livros didáticos (CIRILO, 2008); o estudo hermenêutico sobre a apresentação dos conteúdos no livrotexto (ALVARENGA; PAIXÃO, 2017); e um estudo sobre a proposta de tecnologias digitais nos livros didáticos de Cálculo (RICHIT; RICHIT; FARIAS, 2015).

Por outro lado, nota-se que há uma escassez de estudos com enfoque na visão dos estudantes acerca dos livros didáticos, em particular sobre os livros de Cálculos 
utilizados nos cursos de ensino superior das diversas áreas do conhecimento. Entende-se que uma análise de tais livros, guiando-se pelo olhar dos seus principais usuários, os estudantes da disciplina, proporcionaria material de grande relevância para o planejamento de tarefas relacionadas ao ensino, pesquisa e gestão pelos profissionais que ensinam, elaboram ou são responsáveis pela aquisição de material didático. Além disso, é razoável vislumbrar que uma análise sob essa perspectiva pode colaborar na determinação de mecanismos que subsidiem o professor de Cálculo Diferencial e Integral I na escolha do livro-texto e, também, na elaboração de material didático para as suas aulas, que estejam em sintonia com as expectativas dos alunos.

Diante do exposto, o objetivo deste trabalho consistiu em elaborar um panorama do livro-texto de Cálculo na visão dos alunos que cursaram a disciplina Cálculo I nos cursos de engenharia de uma universidade federal, buscando identificar: aspectos dos livros didáticos que são considerados positivos pelos alunos; o nível de satisfação dos alunos com tais livros apontados nos planos de curso; o nível de influência desse no sucesso ou no fracasso dos alunos que cursaram a disciplina; que materiais didáticos auxiliares são utilizados pelos alunos; o nível de satisfação (sensação de conforto) dos alunos com relação ao uso de livros digitais de Cálculo; e a percepção dos alunos em relação à dependência/independência do professor com o livro adotado.

\section{METODOLOGIA}

A pesquisa desenvolvida neste trabalho pode ser enquadrada como quantitativa, porém aspectos da pesquisa qualitativa também foram considerados, uma vez que se entendeu que um enfoque do estudo baseado nessas duas perspectivas poderia proporcionar uma melhor compreensão do fenômeno estudado. De fato, tais abordagens são complementares e não há contradição entre elas. A qualitativa interpreta os fenômenos à luz do contexto, do tempo e dos fatos; a quantitativa trabalha com técnicas estatísticas na coleta e análise de dados. Trata-se de uma pesquisa de campo, cuja investigação pretendeu analisar um fato social, ou seja, o uso do livro didático na disciplina de Cálculo em curso de engenharia (MICHEL, 2009).

Sendo assim, foram adotados instrumentos de coleta de dados apropriados às duas abordagens, como a aplicação de questionários e análise dos programas de disciplinas, que nos possibilitaram o registro, a análise e a interpretação dos dados.

Primeiramente, foi feito um estudo sobre os livros didáticos utilizados pelos professores de engenharia de uma universidade federal e sobre os livros presentes no Projeto Pedagógico (PPC) de seis cursos de engenharias ofertados. Após isto, foi elaborado um questionário eletrônico estruturado com 22 questões que foi aplicado aos alunos dos cursos de Engenharia Agrícola e Ambiental, Engenharia Civil, Engenharia da Computação, Engenharia de Produção, Engenharia Elétrica e Engenharia Mecânica que faziam parte do universo da pesquisa.

Os sujeitos selecionados foram os estudantes que entraram nos cursos de engenharias nos semestres 2016.1, 2016.2, 2017.1 e 2017.2 que fizeram a disciplina de Cálculo Diferencial e Integral I (aprovados ou reprovados) e que ainda estivessem matriculados no curso. Dessa forma, foram consideradas duas turmas de cada curso de engenharia. 
Conforme dados repassados pela Secretaria de Registro e Controle Acadêmico da instituição (SRCA), relativo aos que ingressaram nos períodos mencionados, e excluindo os alunos que não estavam matriculados, resultou em um universo de 431 discentes.

O questionário eletrônico foi enviado por e-mail para os 431 estudantes selecionados para a pesquisa. Após o prazo de espera para o recolhimento das respostas, foi conseguido um total de 118 questionários respondidos, representando $90 \%$ da amostra ideal. Foram excluídas três respostas de alunos, pois se verificou que esses não cursaram a disciplina de Cálculo I na instituição pesquisada. Isso resultou num total de 115 respostas que foram analisadas.

\section{RESULTADOS E DISCUSSÕES}

Inicialmente, observou-se que, de um modo geral, os títulos presentes nos programas de disciplinas estavam condizentes com os elencados nos PPCs dos cursos, embora tenham sido observados títulos que foram elencados nos PDs, mas que não estavam no PPC e vice-versa. No entanto, o que se pretendia nessa etapa, era identificar quais obras eram mais indicadas pelos docentes para os seus alunos. Esse resultado foi levado em conta na elaboração do questionário dos alunos, que foi a próxima fase da pesquisa.

$\mathrm{Na}$ etapa da análise dos dados, coletados via questionário eletrônico aplicado aos alunos, verificou-se que quatro, dos cinco títulos mais indicados pelos docentes, estavam na relação dos livros preferidos pelos alunos, embora a ordem de preferência não seja exatamente a mesma. Vale destacar a existência de uma obra que foi utilizada por $55,7 \%$ dos alunos, porém não recebeu nenhuma indicação favorável por partes desses.

Algumas perguntas relacionadas à disciplina de Cálculo Diferencial e Integral foram realizadas aos alunos e as respostas obtidas foram reunidas e sumarizadas nos seguintes eventos: aproximadamente 1/4 dos estudantes tiveram dificuldades em ter acesso ao livro, um pouco mais de 1/3 cursaram a disciplina mais de uma vez e aproximadamente $1 / 5$ não seguiram a referência básica indicada pelo professor. Além disso, enfatiza-se a alta porcentagem de alunos que não participaram do programa institucional de monitoria da disciplina, divergindo do estudo realizado por Passos e et al. (2007a), no qual os estudantes indicaram a necessidade de monitoria como uma das sugestões para melhorar seu desempenho.

O uso dos livros sugeridos pelo professor aparece dentre os três recursos mais utilizados pelos discentes, ressaltando a importância desse instrumento para os alunos. Também chama atenção, o número tímido de alunos que usam e-books e livros digitais para estudar a disciplina. Esse resultado contribui para a análise feita por Bode, Khorami e Vissecher (2014), indicando que os alunos precisam ser educados a usar e-books e explorar todos os seus recursos.

Considerando os alunos que aprovaram na disciplina de Cálculo Diferencial e Integral I na primeira vez que cursaram-na, verificou-se que $82 \%$ utilizaram o livro indicado pelo professor. Esse resultado sugere que pode haver uma relação de causa e efeito entre usar o livro adotado pelo professor e ter sucesso nessa disciplina. Apenas $18 \%$ dos alunos que não usaram o livro sugerido pelo professor, conseguiram aprovação cursando-a somente uma vez.

Para analisar a avaliação dos alunos, em relação aos diversos aspectos que compõem o livro didático de Cálculo, foram definidos os seguintes agrupamentos: elementos estruturais, forma de apresentação dos conteúdos, recursos utilizados no aprendizado da disciplina e sugestões para melhorias nos livros. 


\subsection{Elementos estruturais presentes no livro de Cálculo.}

Os elementos avaliados foram agrupados nas seguintes categorias: exemplos, ilustrações e gráficos, demonstração de teoremas, exercícios resolvidos, respostas dos exercícios propostos e existência de problemas contextualizados. O Quadro 1 apresenta o índice de relevância, em porcentagem, atribuído pelos estudantes a cada categoria. Para compor o índice de relevância foram considerados os itens 4 e 5, numa escala de relevância de 1 a 5, onde 1 representa nenhuma relevância e 5 indica total relevância.

Observa-se que a categoria Exemplos é, na visão dos alunos, o elemento de maior relevância nos livros de Cálculo Diferencial e Integral. Pode-se inferir, desse resultado, que a presença de exemplos chaves na elaboração desse recurso didático poderá contribuir para a aprendizagem da disciplina. Ademais, é importante notar a proximidade numérica desse índice com o obtido na categoria Exercícios Resolvidos, indicando que, para os alunos, a existência de exemplos ou exercícios resolvidos é elemento central nesse recurso didático.

\begin{tabular}{|c|c|c|}
\hline \multicolumn{2}{|r|}{ Categoria } & Relevância \\
\hline 1 & Exemplos & $93,9 \%$ \\
\hline 2 & Exercícios resolvidos & $93,0 \%$ \\
\hline 3 & Respostas dos exercícios & $88,0 \%$ \\
\hline 4 & Exercícios de aplicação & $84,3 \%$ \\
\hline 5 & Ilustrações e gráficos & $75,7 \%$ \\
\hline 6 & Demonstrações de teoremas & $55,7 \%$ \\
\hline
\end{tabular}

Fonte: os autores

Para Weiberg (2010), o livro-texto de Cálculo é uma fonte de exercícios para os alunos. Com efeito, os livros costumam apresentar várias seções de exercícios propostos ao longo ou ao final de cada capítulo. Contudo, na visão dos alunos, é importante que as Respostas dos exercícios propostos sejam disponibilizadas.

Na perspectiva dos estudantes, o estudo do Cálculo Diferencial e Integral por meio de uma abordagem contextualizada dos conteúdos também é um elemento que merece atenção nos livros didáticos. Esse resultado também pode estar relacionado ao uso da interdisciplinaridade em tais livros, visto que a aplicação dos conteúdos aos problemas de engenharia vai além da prática de contextualização. Esse resultado corrobora a análise de Alvarenga e Paixão (2017, p.166) que observaram que autores do livro-texto analisado "perdem a oportunidade de fornecer aos leitores maior desenvolvimento procedimental e interdisciplinar dentro da própria matemática."

A presença de ilustrações e gráficos é outro item relevante para os alunos. Esse resultado vai ao encontro do obtido por Richit, Richit e Farias (2015), que apontaram para a necessidade de uma abordagem de Cálculo Diferencial e Integral que propicie uma compreensão mais integral dos conteúdos por meio de múltiplas representações (contextos algébricos, gráficos e numéricos).

\section{Maneira como os conteúdos básicos estão apresentados nos livros}


Foi solicitado aos alunos que avaliassem como os conteúdos básicos de Cálculo I (limites, continuidade, derivada e integral) estão apresentados nos livros utilizados. Os alunos atribuíram notas de 1 a 5 , onde o número 1 indica que o tema está muito mal apresentado, enquanto que o 5 indica que o tema está muito bem apresentado. Para fins de análise, as duas primeiras notas foram agrupadas na categoria Avaliação negativa e as duas últimas na categoria Avaliação positiva. A nota intermediária foi registrada na categoria Avaliação média. Os resultados estão apresentados no Quadro 2.

Quadro 2 - Avaliação dos conteúdos nos livros de Cálculo
\begin{tabular}{|c|c|c|c|}
\hline Conteúdo & $\begin{array}{c}\text { Avaliação } \\
\text { Negativa }\end{array}$ & $\begin{array}{c}\text { Avaliação } \\
\text { média }\end{array}$ & $\begin{array}{c}\text { Avaliação } \\
\text { Positiva }\end{array}$ \\
\hline Limites & $11,3 \%$ & $40,9 \%$ & $47,8 \%$ \\
\hline Continuidade & $17,4 \%$ & $51,3 \%$ & $31,3 \%$ \\
\hline Derivadas & $11,3 \%$ & $33,0 \%$ & $55,7 \%$ \\
\hline Integrais & $8,7 \%$ & $39,1 \%$ & $52,2 \%$ \\
\hline
\end{tabular}

Fonte: os autores

De um modo geral, a maioria dos alunos avaliou que os conteúdos estão bem apresentados ou forma mediana. Os conteúdos derivadas e integrais foram considerados os mais bem apresentados. Importante destacar que o conteúdo continuidade de funções recebeu o maior índice de avaliação negativa e o menor índice de avaliação positiva.

\section{Recursos didáticos para o aprendizado do Cálculo.}

Pediu-se que os estudantes avaliassem, numa escala de 1 a 5, a contribuição de alguns recursos didáticos para o melhor aprendizado na disciplina de Cálculo I. O resultado está apresentado no Quadro 3, onde o índice de relevância foi calculado considerando as notas 4 e 5.

\begin{tabular}{|c|c|c|}
\hline \multirow{2}{*}{\multicolumn{2}{|c|}{ Recurso }} & \multirow{3}{*}{$\begin{array}{c}\text { Relevância } \\
71,3 \%\end{array}$} \\
\hline & & \\
\hline 1 & Livro didático & \\
\hline 2 & Linguagem do livro didático & $81,7 \%$ \\
\hline 3 & Sequência dos conteúdos & $60,9 \%$ \\
\hline 4 & Monitoria de Cálculo I & $53,0 \%$ \\
\hline 5 & Videoaulas & $78,3 \%$ \\
\hline
\end{tabular}

Fonte: os autores

Observa-se que a linguagem do livro de cálculo, o livro e videoaulas foram os itens apontados como mais relevantes para o aprendizado da disciplina. $O$ resultado apresentado na Categoria 4, Quadro 3, merece uma reflexão, pois apesar de $53 \%$ dos 
alunos atribuírem os níveis mais altos de relevância à atividade Monitoria, apenas 27,8\% afirmaram ter participado do programa de monitoria ofertado pela instituição, contrariando Passos et al. (2007), em que os alunos apontaram a falta de monitoria como uma das causas do baixo rendimento, mas não aproveitaram a oportunidade.

\section{Indicação de melhorarias nos livros de Cálculos.}

O Quadro 4 apresenta os elementos dos livros que deveriam ser melhorados na visão dos alunos e os respectivos índices de relevância atribuídos.

\begin{tabular}{|c|c|c|}
\hline \multicolumn{2}{|r|}{ Categoria } & Relevância \\
\hline 1 & A quantidade de exemplos & $36,5 \%$ \\
\hline 2 & $\begin{array}{l}\text { A quantidade e qualidade das } \\
\text { ilustrações }\end{array}$ & $19,1 \%$ \\
\hline 3 & A quantidade de exercícios resolvidos & $56,5 \%$ \\
\hline 4 & As demonstrações dos teoremas & $37,4 \%$ \\
\hline 5 & Respostas dos exercícios propostos & $53,9 \%$ \\
\hline 6 & A quantidade de exercícios propostos & $13 \%$ \\
\hline 7 & A linguagem utilizada & $61,7 \%$ \\
\hline
\end{tabular}

Fonte: os autores

Segundo os alunos, a linguagem dos livros de cálculos deve ser melhorada. Ademais, a quantidade de exemplos, de exercícios resolvidos e respostas aos exercícios propostos obtiveram índices elevados de necessidade de melhorias. Ainda sobre exercícios propostos, o conjunto de dados, apresentados no Gráfico 1, indica que os alunos consideram que exercícios com resolução de problemas práticos contribuem para o seu aprendizado da disciplina. Esse resultado vai ao encontro dos resultados obtidos por Alvarenga e Paixão (2017), que questionam a ausência de atividades interdisciplinares no livro de cálculo; e por Richit, Richit e Farias (2015) que destacam a necessidade de os livros didáticos fomentarem novos modos de ensinar e aprender.

Os alunos também foram questionados sobre a sensação de conforto no uso de livros digitais e e-books. Nesse quesito, $61 \%$ dos discentes se sentem confortáveis utilizando-os, enquanto $36 \%$ sentem desconforto em ler nesse tipo de material. Apenas $3 \%$ dizem que não utilizam livros digitais. Importante relacionar esse dado com o obtido no estudo realizado por Bode, Khorami e Vissecher (2014), sendo que apenas $41 \%$ dos alunos afirmaram ter uma experiência muito positiva com o uso de e-book de Cálculo.

Por fim, pediu-se aos alunos que escrevessem livremente uma mensagem que achassem pertinente sobre Cálculo I e o livro didático. A análise das respostas sugerem três agrupamentos: Críticas à linguagem, às metodologias e práticas pedagógicas dos docentes, à quantidade e qualidade dos exemplos e exercícios resolvidos. Dentre as respostas apresentadas, analisa-se que duas sintetizam a visão dos alunos sobre a disciplina e os livros-textos. Mensagem 1: "Cálculo 1 é o primeiro contato com situações, teoremas e aplicações no nível universitário, pelo menos pra mim foi dos cálculos o que 
mais demandou horas de revisão e entendimento de teoremas e exercícios, em comparação com C2 e C3. Uma gama maior de exercícios que abordagem de maneira detalhada as possíveis situações das questões, tal como demostrações claras dos teoremas, poderiam colaborar para a melhora no entendimento do assunto". Mensagem 2: "Não consigo estudar por livros. Tenho dificuldade com a linguagem apresentada e interpretação do conteúdo. Estudo apenas por videoaulas e exercícios ministrados pelo professor."

Gráfico 1 - Tipo de exercícios que contribuem para o aprendizado

18- Qual tipo de exercicio você acha que contribui mais para o teu aprendizado?

115 respostas

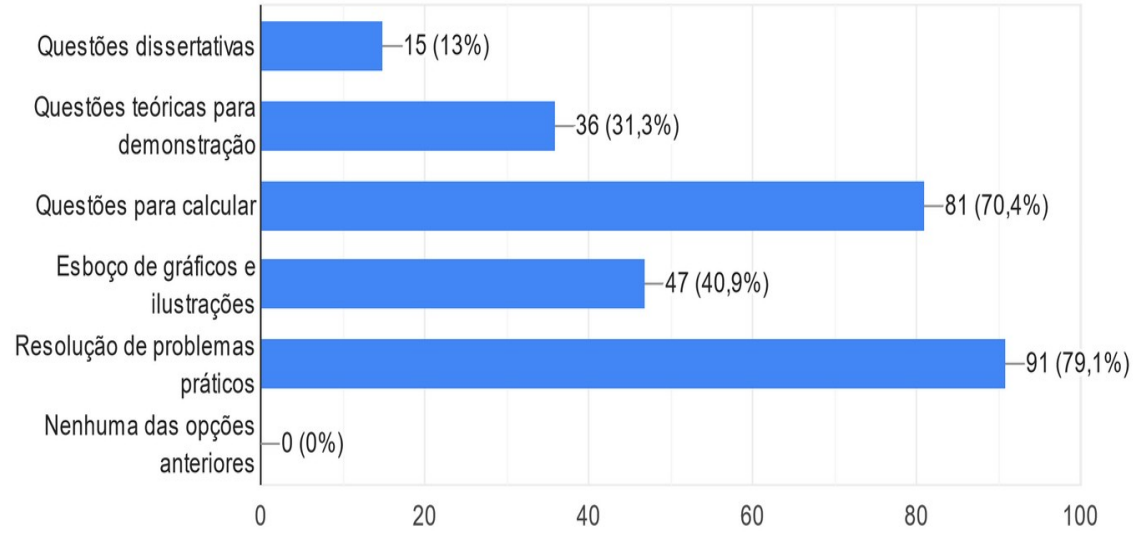

Fonte: os autores

As sugestões apontadas pelos alunos parecem ir ao encontro dos resultados observados por Weiberg(2010) considerando que para ajudar os estudantes a verem os livros textos como um meio útil de aprender matemática, os docentes precisam entender que ferramentas e características um estudante precisa, para ler com sucesso um livrotexto da disciplina.

\section{CONSIDERAÇÕES FINAIS}

De modo geral, a escolha e elaboração de materiais didáticos são realizadas com base na percepção do docente e sua prática pedagógica. Dessa forma é possível que não estejam contemplados, nessa escolha, quesitos que são relevantes para o aprendizado dos alunos. Assim, este trabalho pretendeu apresentar um panorama que possa auxiliar o professor durante a preparação de material didático e na escolha do livro didático de Cálculo Diferencial e Integral.

Os objetivos foram alcançados e os resultados apontaram que o livro didático e a clareza da linguagem utilizada pelos autores são elementos muito relevantes para o aprendizado da disciplina. No entanto, no que diz respeito às contribuições dos elementos estruturais do livro, os discentes ressaltaram exemplos e exercícios resolvidos como mais significativos. 
No caso específico dos exercícios propostos, os alunos destacaram as questões de calcular e de aplicação dos conteúdos em detrimento das questões de esboçar gráficos ou fazer ilustrações.

De um modo geral, para eles, os conteúdos Derivadas e Integrais são os melhores apresentados nos livros e sugerem melhorias na linguagem, na quantidade de exemplos, de exercícios resolvidos e respostas aos exercícios propostos.

Com relação aos recursos de apoio à aprendizagem, constatou-se que o uso do livro didático sugerido pelo professor, a estratégia de refazer exercícios resolvidos e assistir videoaulas são os mais utilizados pelos estudantes de Cálculo.

Pôde-se inferir ainda que o uso do livro didático, indicado pelo professor, pode ter uma influência direta na aprovação da disciplina de Cálculo Diferencial e Integral, contudo, o estabelecimento dessa relação necessita de um estudo mais aprofundado, que será realizado futuramente.

\section{Agradecimentos}

Agradecemos ao CNPq pelo apoio financeiro da bolsista de iniciação científica.

\section{REFERÊNCIAS}

ÁVILA, Geraldo. O Ensino do Cálculo e da Análise. Revista Matemática Universitária, n.33, p.83-95, 2002.

Disponível

em:

https://rmu.sbm.org.br/wp-content/uploads/sites/27/2018/03/n33_Artigo05.pdf.

Acesso

em: 8 abr. 2021.

ALVARENGA, Karly B; PAIXÃO, Aline da. Hermenêutica: análise de um livro didático de cálculo diferencial e integral. Acta Latinoamericana de Matemática Educativa, v.30, p.158-167, 2017. Disponível em: http:// www.clame.org.mx. Acesso em: 08 abr. 2021.

BODE, Martina; KHORAMI, Mehdi; VISSCHER, Daniel. On the effectiveness of Calculus e-books. In: 26th International Conference on Technology in Collegiate Mathematics ICTCM-26 , 2014,San Antonio. Anais. 2014.

Disponível em: http://archives.math.utk.edu//CTCM/i/26/S095.html. Acesso em: 08 abr. 2021

CIRILO, Kassiana S. S. Livros Didáticos e Modelagem Matemática: uma caracterização da Transposição Didática do Conteúdo de Integral nestes ambientes. Dissertação (Mestrado em Ensino de Ciências e Educação Matemática). Londrina: Universidade Estadual de Londrina, 2008.

CURI, Rosires C; FARIAS, Raliny M.S. Métodos de estudo e sua influência no desempenho dos alunos em disciplinas de cálculo diferencial e integral. In: CONGRESSO BRASILEIRO DE EDUCAÇÃO EM ENGENHARIA, XXXVI, 2008. Anais. São Paulo, 2008.

Disponível em: http://www.abenge.org.br/cobenge/legado/arquivos/11/artigos/2448.pdf. Acesso em: 29 abr. 2021. 
MATEUS, Pedro. Cálculo diferencial e integral nos livros didáticos: uma análise do ponto de vista da organização praxeológica. Dissertação (Mestrado em Educação). São Paulo: Pontifícia Universidade Católica de São Paulo, 2007.

MENDES, Karina B; GIOSTRI, Elza C. O ensino de cálculo I e a realidade dos alunos de engenharia e tecnologia. In: CONGRESSO BRASILEIRO DE EDUCAÇÃO EM ENGENHARIA, 2008. Anais. São Paulo, 2008.

Disponível em: http://www.abenge.org.br/cobenge/legado/arquivos/11/artigos/3449.pdf. Acesso em: 29 abr. 2021.

MOREIRA, H; CALEFFE, L. G. Metodologia da pesquisa para o professor pesquisador. Rio de janeiro: DP\&A, 2006

MICHEL, Maria Helena. Metodologia e pesquisa científica em ciências sociais. 2. ed. atual. e ampl. São Paulo: Atlas, 2009.

THIOLLENT, Michel. Metodologia da pesquisa-ação. 5. ed. São Paulo: Cortez, Autores Associados, 1992.

PASSOS, Fabiana G; VICHI, Cristian; DUARTE, Francisco R; SOUSA, Geida M. C; TELES, Renato S; SANTOS, Vivianni M. L. Diagnóstico sobre a reprovação nas disciplinas básicas dos cursos de engenharia da Univasf. In: Congresso Brasileiro de Educação em Engenharia, XXXV, 2007. Anais. Curitiba, 2007.

Disponível em: http://www.abenge.org.br/cobenge/legado/arquivos/12/artigos/298-Fabiana \%20dos\%20Passos.pdf. Acesso em: 29 abr. 2007.

PASSOS, Fabiana G; DUARTE, Francisco R; LEITE, Ângelo A.M; PEREIRA, Paulo J; LEITE, Télio N; DONZELI, Vanessa P. Análise dos índices de reprovações nas disciplinas cálculo I e geometria analítica nos cursos de engenharia da UnivasF . In: CONGRESSO BRASILEIRO DE EDUCAÇÃO EM ENGENHARIA, XXXV, 2007. Anais. Curitiba, 2007. Disponível em: http://www.abenge.org.br/cobenge/legado/arquivos/12/artigos/199-Fabiana \%20dos\%20Passos.pdf. Acesso em: 29 abr. 2021.

RICHIT, Andriceli; RICHIT, Adriana; FARIAS, M.M.R. Cálculo Diferencial e Integral e Tecnologias Digitais: o que propõem os livros didáticos de Cálculo? In: XIV CIAEMIACME, 2015. Anais. Chiapas, 2015.

Disponível em: http://xiv.ciaem-redumate.org/index.php/xiv_ciaem/xiv_ciaem/. Acesso em: 08 abr. 2021.

WEINBERG, Aaron. How Students Use Their Textbooks: Reading Models and Model Readers. In: BROSNAR, p., ERCHICK, D.B., \& FLEVARES, L. (Eds.). PROCEEDINGS OF THE 32ND ANNUAL MEETING OF THE NORTH AMERICAN CHAPTER OF THE INTERNATIONAL GROUP FOR PSYCOLOGY OF MATHEMATICS EDUCATION.v. 6. Columbus: The Ohio State University, 2010, p. 105-113. 
PERCEPTIONS OF ENGINEERING STUDENTS ABOUT THE DIFFERENTIAL AND INTEGRAL CALCULUS BOOK

\begin{abstract}
The textbook is an important tool in the teaching and learning process of a discipline. In particular, in the Differential and Integral Calculus discipline, this instrument has great success for being an important interlocutor between the content of the discipline and the student. However, it is known that the choice of textbooks is, in general, made only from the perspective of the instructors. In this way, it is possible that elements valued by students and that contributes to their learning curve are not taken into account in the textbooks. In this work, we used a field research, of qualitative and quantitative nature, whose investigation intended to analyze a social fact, the use of the Calculus textbook adopted in engineering courses, with a view to elaborating a panorama of the book in the view of the students who took a course at a Federal University. The main results pointed out that the Calculus textbook can have a direct influence on the approval of students in the discipline and the clarity of the language used by the authors is a very relevant element for students learning curve. Beside that, the students suggest improvements in the language, in the number of examples, in application exercises, solved exercises and responses to the exercises proposed in the textbooks.
\end{abstract}

Keywords: Mathematics Teaching, Differential and Integral Calculus, Textbook. 